\title{
ABSTRACT \\ Efficacy of duloxetine in addition to self-management strategies for treatment of chronic paiful temporomandibular disorder: a randomized, placebo-controlled clinical trial
}

Rigorous evidence for combining different therapies for chronic painful temporomandibular disorder (TMD) is limited. Therefore, we conducted a randomized, double-blind, placebo-controlled trial 1) to assess the efficacy of duloxetine in addition to self-management (SM) strategies for treatment of chronic TMD; 2) to investigate whether baseline conditioned pain modulation (CPM) predicts the efficacy of duloxetine in TMD individuals; and 3) to conduct an exploratory analysis of five phenotyping domains - pain, psychological, sleep, quantitative sensory testing and CPM - to examine predictors of response to SM-duloxetine. Participants were randomized 1:1 to duloxetine $60 \mathrm{mg}$ or placebo once daily for 12 weeks. Moreover, all participants were treated with a SM program. The primary outcomes were a) the change in the pain intensity from baseline to week 12 and b) CPM-sequential paradigm at baseline. Supplemental pain measures, physical and emotional functioning outcomes were also evaluated. Modified baseline observation carried forward, ANCOVA, multiple linear regression and relative risk were applied to the data $(\mathrm{p}<0.050)$. Eighty participants were randomized and 78 were included in the intention-to-treat analysis. Pain intensity decreased significantly over time with participants on SMduloxetine and SM-placebo, reporting reductions from baseline of $30 \%$ and $36 \%$, respectively, but did not differ significantly between groups $(0.3,95 \% \mathrm{CI}:-1.1,1.7 ; \mathrm{p}=0.82)$. A more efficient CPM was associated with a greater pain intensity reduction $(\mathrm{p}=0.035)$ after 12 weeks of treatment, regardless the treatment group. Furthermore, phenotypes, e.g., severe pain intensity, pain disability, painful comorbidity and anxiety symptoms were indicative of the likelihood of response to SM-duloxetine. In conclusion, there is no beneficial effect of adding duloxetine to SM strategies for treatment of chronic TMD, although high attrition and confidence interval interpretation preclude firm conclusions. Moreover, this randomized clinical trial demonstrated the feasibility of applying patient phenotyping assessment to predict short-term treatment response in chronic TMD individuals, which can contribute to the development of mechanism-based treatments of orofacial pain.

Keywords: Temporomandibular joint dysfunction syndrome. Chronic pain. Duloxetine hydrochloride. Self-care. Pain threshold. Randomized controlled trial 